
\title{
R Reserach S Suare \\ Filler Dependent Microstructural and Optical Properties of PVDF/PEG Blends Versus PVDF/GO Nanocomposites
}

Maheswar Panda ( $\sim$ panda.maheswar@gmail.com )

Dr. Harisingh Gour Vishwavidyalaya (A Central University) https://orcid.org/0000-0002-0711-4647

\section{Research Article}

Keywords: composites, blends, dispersions, optical properties, spectroscopic ellipsometry

Posted Date: November 3rd, 2021

DOI: https://doi.org/10.21203/rs.3.rs-1039145/v1

License: (c) (1) This work is licensed under a Creative Commons Attribution 4.0 International License.

Read Full License 


\section{Abstract}

The micro-structural features and the optical properties of polymer blends (PB) of polyvinylidene fluoride (PVDF) base with polyethylene glycol (PEG) and polymer nanocomposites (PNC) of PVDF base with graphene oxide (GO) as fillers are compared. Different weight fractions of PVDF/PEG and PVDF/GO samples were prepared through solution casting followed by spin coating. The microstructure of PVDF/PEG and PVDF/GO shows formation of immiscible/compatible/ homogenous microstructures over different extents of loading of the filler components. UV-Visible spectrometry revealed an increase in absorbance with the amount of filler and the absorbance was found to be lesser and highly dispersive for the PB as compared to PNC, attributed to the heterogeneity/electrical conductivity of the respective fillers of the two systems. Spectroscopic ellipsometry was used to study the various optical constants (e.g. refractive index, extinction co-efficient, etc.) \& their dispersion behavior over the visible range of wavelengths. The parameters show large variation as a function of component fillers due to the different extent of interaction of matrix/filler. The optimized parameters were obtained for the $20 \%$ PB \& the $5 \%$ PNC samples respectively, suggesting PNC with nano fillers are always to be a better choice for developing them as materials suitable for various optical applications.

\section{Introduction.}

Polymer nano composites [PNC] are found to be very important materials in recent years because of their large number of applications in various fields, such as; polymer dielectrics, polymer ferroelectrics, polymer piezoelectrics, polymer magneto-electrics, polymer magneto-dielectrics, flexible optical/optoelectronics materials, etc. [1-6]. These materials are finding interest due to their possible commercial applications in sensors, actuators, transducers, capacitors, memory devices, etc. The importance of these materials is mainly due to the complex interfacial region between the filler/polymer giving rise to tremendous variation in their intended physical properties required for various multifunctional applications, due to the large surface area of the fillers. They have become very smart materials from the point of energy storage [7-13], ferroelectrics [14] and multiferroics/magnetoelectrics [15-17], magnetodilectrics, [18], optical/optoelectronics [19-24], biomedical [25] or any other multifunctional applications, due to their inherent flexibility solving the most complicated designing problems. The various types of nano fillers used in these PNC are e.g. metal, carbon black, CNT, ceramics, graphene and graphene derivatives, various types of carbon nanostructures, etc. [1-25]. In the development of polymer dielectrics/ferroelectrics, the varieties of metal and ferroelectric ceramic nano particles have been introduced into the PVDF matrix [7-13]. In development of polymer multiferroics/magnteoelectrics/magneto-dielectrics, the particulate composites based on various fillers have been introduced [15-18]. Similarly in the development of PNC for optical/optoelectronic applications, metal nanofillers and various types of carbon nanostructures, are introduced to the polymer matrices [19-26]. In preparation of these PNC, the ferroelectric polymers, e.g. polyvinylidene Fluoride (PVDF), poly[(vinylidene-difluoride-trifluoroethylene] [P(VDF-TrFE)], etc. are always taken as preferred 
polymers for developing them for various applications with the preferred fillers of various types of carbon nanostructures, such as graphene oxide (GO), carbon nanotube (CNT), etc. [1-26].

However, it is always interesting to observe the other ways/ process of development of these types of optical materials, out of which one process is forming the blends of one polymer with the other polymers, e.g. ferroelectric polymers [e. g. PVDF] to form polymer blends with other polymers, which are under rapid development for finding their suitability for optical applications [27-32]. It is always interesting to introduce novel types of polymers with higher optical activity into the polymer matrix such as PVDF matrix by novel synthesis method to produce highly homogenous thin films to have enhanced optical properties. Since, polyethylene glycol (PEG) is a flexible, non-toxic, hydrophilic polymer with higher optical activity, the blends of PVDF with PEG are expected to show better optical properties.

Hence, with the aim of development of PB/PNC flexible material with better optical properties based on PVDF matrix, the comparison of the optical properties of PVDF/PEG PB versus PVDF/GO PNC have been undertaken as a case study with their respective innovative combinations/compatibility to find their optical performances. The results show interesting homogeneous/heterogeneous microstructures formed from their combinations over different extent of the loading of their component fillers. Interesting optical properties in terms of absorbance, psi/delta [storage/loss/phase variation], refractive index, extension coefficient, real/imaginary dielectric constants, in the region of visible wavelengths are explored and are compared for finding their suitability for optical applications.

\section{Experimental Details.}

GO was prepared through the modified Hummar's method [33], and the details of the preparation are given in the recently published article [24]. One series of PB based on PVDF with varying weight fractions of PEG from 0.0 to 0.4 [Table 1] and PNC based on PVDF with varying weight fractions of GO from 0.0 to 0.2 were prepared [Table 2]. The PVDF/PEG blends and PVDF/GO nanocomposites were prepared through the two steps of solution casting followed by spin coating. In order to prepare the thin film, a square shaped glass substrate is fixed on the spin arm of the spin coater by high suction pump and a small amount of a solution is applied to the substrate by a micro pipette, which is then rotated at high speed for 20 seconds in order to spread it using centrifugal forces, making a dry thin film available on the substrate. The details of sample preparation and experimental characterizations can be found from the recent reference [24]. The microstructure investigation was carried out using Thermo Fisher Nova SEM 450. The UV-Visible spectroscopic measurements were made with the help of Thermo Fisher Evolution 301 in the wavelength range of $400 \mathrm{~nm}$ to $700 \mathrm{~nm}$. The ellipsometric measurements were carried out with the help of J.A. Wollam M-2000 spectroscopic ellipsometer.

\section{Results And Discussion.}

\subsection{Microstructure}


The FESEM micrographs of the pure PVDF [Fig.1 (a)] and PVDF/PEG PB with varying weight fractions of 0.2 and 0.4 are shown in Figure $1(b-d)$. The large created unfilled like structures and higher heterogeneity and immiscibility is clearly observable from the micrographs for the sample 0.2 [Fig.1 (b)], while for PB with 0.4 of PEG becomes more compatible and homogenous [Fig.1 (c) \& (d)], suggesting the homogenousness of PB at higher loadings of PEG. The diameter of the created structures in the PB, are of the order of $\sim 0.1 \mu \mathrm{m}$. From these micrographs of PVDF/PEG PB, it is clear that various types of interfaces have appeared in the blends, which can result in an increase of the absorbance. The FESEM images of PVDF/GO composites with different weight fractions $0.02,0.05,0.10,0.20$ are shown in the Figure 1 (e-h). All these micrographs of PVDF/GO nanocomposites show excellent homogenous microstructures of uniformly dispersed GO of the order of $1 \mu \mathrm{m}$ range. With the increase of loading of GO, the homogeneity increases from 0.0 to $0.05[\mathrm{Fig.1}(\mathrm{e}-\mathrm{f})]$ and then the homogeneity decreases from 0.05 to 0.2 , resulting in the agglorameration of GO [Fig.1 (g-h)]. This also clearly indicates that on addition of GO, that may result to increase in absorbance of these PNC, due to higher conductivity of GO.

\subsection{UV-Visible Spectroscopy}

The UV-visible spectroscopy is one of the methods of finding the optical activity/response of the samples by collecting the information of absorbance/transmittance/reflectance of the samples in the ultraviolet-visible spectral region. Generally, the light in the visible and adjacent [near-UV and nearinfrared] ranges of wavelengths are passed through the samples. The light interacts with the samples [some possible interactions are molecules containing $\pi$-electrons or non-bonding electrons can absorb the energy in the form of ultraviolet or visible light to excite these electrons to higher anti-bonding molecular orbitals]. The result of light/matter interaction are represented in terms of various optical parameters, such as; absorbance/transmittance/reflectance as a function of wavelength in the ultraviolet-visible spectral region of interest. The more easily the electrons are excited, the longer is the wavelength of light that it can absorb. When light passes through or is reflected from a sample, the amount of light absorbed is the difference between the incident radiation $\left(I_{0}\right)$ and the transmitted radiation ( () . The amount of light absorbed is expressed as either transmittance or absorbance. Transmittance usually is given in terms of a fraction/as a percentage and is defined as

$$
T=\frac{I}{I_{0}} \text { or } \% T=\frac{I}{I_{0}} * 100 \text {. }
$$

. The absorbance is related to the transmittance and is defined as

$\alpha=-\log T$, which is given in the Figure 2. Figure 2 shows the UV-Visible absorbance spectra of both the series of samples in the visible region of wavelength range of $400 \mathrm{~nm}$ to $700 \mathrm{~nm}$ of light. There is an increase of absorbance linearly with increase of PEG into the PB, and the absorbance reaches maximum as 0.25 for 0.4 [Fig. 2 (a)]. However, for all the PB, the absorbance is highly dispersive in nature, suggesting as well attributed to the structure of PB to be highly heterogeneous and incompatible, confirmed from the Fig. 1 (a-d). A continuous decrease of absorbance with increasing wavelength up to $550 \mathrm{~nm}$ is also clearly visible. In a comparison to the PNC, the increase of absorbance also occurs linearly with increase of GO into the PNC, and the absorbance remains constant and nondispersive over whole visible region of wavelength range of $400 \mathrm{~nm}$ to $700 \mathrm{~nm}$ of light. From graph [Fig. 2 (b)], it is observed 
that the absorbance of pure PVDF solution (black line) decreases with wavelength from approximately 0.2 at $400 \mathrm{~nm}$ to 0.04 at $700 \mathrm{~nm}$. But with the increase of $\mathrm{wt} \%$ of GO in the PNC, the absorbance approaches as high as 3.5 at $400 \mathrm{~nm}$ to 3.0 value at $700 \mathrm{~nm}$ for the PNC with $10 \% \mathrm{GO}$. It shows the distinct curves for different loadings of fillers of PB/ PNC and the absorbance decreases with wavelength in a systematic manner from higher to lower value with decreasing wt\% of the fillers. The absorbance curves for PB are stiffer as compared to the PNC, attributed to their heterogeneity, confirmed from the microstructures [Fig. 1].

The increase in absorbance in both the cases, with increase of filler amount can be attributed to the electrical conductivity of the filler. Also, the higher conductivity of GO as compared to the PEG, is responsible for giving more than 10 times higher absorbance in case of PNC as compared to PB. The exclusive micro structure of both the series of samples confirms these interesting consequences also [Fig. 1].

\subsection{Spectroscopic Ellipsometric Studies}

Spectroscopic ellipsometery was used to study the variation of the optical parameters of the thin films of $\mathrm{PB}$ and PNC [30-32]. The SE is a high precise and nondestructive powerful tool to extract the varius optical parameters of the films with accurately and reliably results. Ellipsometry (reflection ellipsometry) measures the changes in the state of polarization of light upon reflection from a surface. As a noninvasive and non-destructive tool, ellipsometry requires only a low-power light source and, consequently, it does not affect most processes, which renders ellipsometry a convenient tool for in situ studies. Figure 3 show the plot of ellipsometric parameters, psi $(\psi)$ and delta $(\Delta)$ versus wavelength $(\lambda)$ in the visible wavelength region from $500 \mathrm{~nm}$ to $800 \mathrm{~nm}$ for all the PB/PNC under investigation. The significant variation in the ellipsometric parameters $\psi$ and $\Delta$ for all the PB/PNC confirms the significant change in the amplitude as well as phase ratio of $S$ and $P$ polarized light. Further the extent of variation of $\Psi$ and $\Delta$ for all the PB samples with $0.0,0.10,0.3$ and 0.4 is highly varying, while for the PB with 0.2 , the $\Psi$ and $\Delta$ remains almost constant throughout the wavelengths [Fig. 3 (a-b)]. However the extent of variation of $\Psi$ and $\Delta$ for all the PNC samples with $0.0,0.01,0.02,0.05$ are remaining constant, while for the samples with higher loading of GO, i.e. for 0.1 and 0.2 , the $\Psi$ and $\Delta$ are highly varying throughout the wavelengths [Fig. 3 (c-d)]. The differences of these optical features are attributed to the homogenity of the samples and the differences of the electrical conductivity of their respective fillers. I found that the PB sample with $20 \%$ of PEG and PNC sample with $5 \%$ GO, show same value of ellipsometric parameters $(\Psi \sim 30$ and $\Delta \sim 5)$ and these values also remains constant over the whole wavelength range. Thus these optical parameters also demand that the $20 \%$ of PEG in the PB and $5 \%$ of GO in the PNC need to be highly homogenous, and these can be also well confirmed from the highly homogenous microstructures respectively [Fig. 1 ( $\mathrm{b} \& \mathrm{f}$ )]. Thus the extent of variation in parameters $\psi$ and $\Delta$ confirms the homogeneity and the morphological changes of the PB/PNC, and find their suitability for optical applications.

The variation of real and imaginary parts of complex refractive index ( PB/PNC, where ' $n$ ' and ' $k$ ' represents the refractive index and the extinction coefficient of the PB/PNC 
films with wavelengths in the range $500 \mathrm{~nm}$ to $800 \mathrm{~nm}$. It can be observed that there is a significant change in the refractive index and extinction coefficient for all PB/PNC samples with weight \% of the filers in the PB/PNC over the wavelengths in the range of $500 \mathrm{~nm}$ to $800 \mathrm{~nm}$. Similar type of observation as that of variation of $\psi$ and $\Delta$ was found for ' $n$ ' and ' $k$ ' for both PB/PNC samples. In the case of PB, $10 \% \& 20 \%$ of PEG in the PB sample, show higher value of refractive index [1.1-1.3] with low extinction coefficient [0.0-0.05] was observed and are found to be constant in the region of wavelength of $500 \mathrm{~nm}$ to $800 \mathrm{~nm}[\mathrm{Fig} .4(\mathrm{a}-\mathrm{b})]$. However for the PNC with wt\% of GO as $0.02,0.03$ and 0.05 , higher value of refractive index [1.2-1.3] with low extinction coefficient [0.0-0.1] was observed and are found to be constant in the region of wavelength of $500 \mathrm{~nm}$ to $800 \mathrm{~nm}$ [Fig. 4(c-d)]. Hence these respective fluctuations and constancy of the optical parameters can be attributed to the extent of heterogeneity/homogeneity of the PB/PNC samples respectively [Fig. 1].

Similarly, the variation of real and imaginary parts of complex dielectric function $\left(\varepsilon=\varepsilon^{\prime}+i \varepsilon\right.$ ") [Fig. 5] are shown for all the PB/PNC, where ' $\varepsilon$ ' and ' $\varepsilon$ '" represents the real part and the imaginary parts of the complex dielectric function of the PB/PNC films with wavelengths in the ranges of $500 \mathrm{~nm}$ to $800 \mathrm{~nm}$. From figure 5, the similar types of observations were recorded for both PB/PNC as that of Figure 3 and 4 . The real and imaginary parts of complex dielectric function remains constant for the samples with wt\% as 0.20 , while large variations were obtained for all other PB. Similarly, the real and imaginary parts of complex dielectric function remains constant for the samples with wt $\%$ as 0.03 and 0.05 , while large variations were obtained for all other PNC. From all the graphs [Fig. 3 to Fig. 5], it can be observed that all the optical parameters of the PB sample with $20 \mathrm{wt} \%$ of PEG and for PNC sample with GO as 0.05 , are highly stable over the complete wave length region and these achieved stabilized values are also attributed to the excellent homogeneity of the samples as prepared [Fig. 1 (b) and Fig. 1(f)].

\section{Conclusions}

In conclusion, the micro structural and optical properties of PVDF/PEG PB and PVDF/GO PNC prepared through spin coating technique have been studied. The effects of the two fillers on the structural and optical properties like absorbance, refractive index, extinction coefficient real and imaginary parts of complex dielectric function etc. were investigated. The UV-Visible absorbance spectra of both PB/PNC samples revealed an increase in absorbance with addition of fillers. Further, the value of absorbance with $5 \%$ PNC was nearly 10 times higher than that of $40 \% \mathrm{~PB}$, attributed to the higher electrical conductivity of GO. From this it can be concluded that PVDF/GO PNC are more optically active in comparison to PVDF/PEG PB. Similarly, the elipsometric parameters were also found to be dependent on the loading of the fillers in the case of both PB and PNC. The optical parameters of the PB sample with $20 \mathrm{wt} \%$ of PEG and for PNC sample with $\mathrm{GO}$ as $5 \%$, are highly stable over the complete wave length region and also are of the same order of the value for all optical parameters. Hence on the basis of flexibility, homogeneity and optical activity, the two samples, i.e. 20wt\% of PEG among all the PB samples and 5\% of GO among all the PNC samples are going to be the effective materials for optical/optoelectronic applications. Even if more specifically, by looking at the observed excellent homogeneity, uniformity of absorbance \& other 
optical parameters, the $5 \%$ of GO PNC is the comparatively better material developed for optical/optoelectronic applications.

\section{Declarations}

Acknowledgements: The author acknowledges Mr. Soumitra Das for preparing the samples and the Sophisticated Instrumentation Centre, Dr. Harisingh Gour Vishwavidyalaya for providing the experimental data.

Conflict of Interest: The author declares that they have no conflict of interest.

\section{References}

1. Dang, Z.M.: Dielectric Polymer Materials for High-Density Energy Storage. Elsevier, Amsterdam (2018)

2. Huang, X., Zhi, C.: Polymer Nanocomposites: Electrical and Thermal Properties. Switzerland, Springer (2016)

3. Hussain, C.M., Mishra, A.K.: (2018). New Polymer Nanocomposites for Environmental Remediation. Netherlands, Elsevier

4. Uchino, K.: (2017). Chapter 1: The Development of Piezoelectric Materials and the New Perspective, Advanced Piezoelectric Materials (Second Edition), Woodhead Publishing

5. Szabó, D.V.: (2017). Chapter 17: Polymer Nanocomposites for Optical Applications; in the book Advances in Polymer Nanocomposites, Woodhead Publishing. Pp 567-604

6. Koo, J.H.: Chapter 12: Optical Properties of Polymer Nanocomposites, Fundamentals, Properties, and Applications of Polymer Nanocomposites. Cambridge University Press (2017)

7. Panda, M.,: (2021). Tuned dielectric and percolation behavior of cold pressed polyvinyledene fluoride nanocomposites caused by $\mathrm{Ni}$ and $\mathrm{BaTiO}_{3}$ filler. Ind. J. Phy. DOI:10.1007/s12648-021-02093-2

8. Panda, M., Mishra, A., and Shukla, P.: Effective enhancement of dielectric properties in cold pressed polyvinyledene fluoride/Barium Titanate nanocomposites. SN Appl. Sci. 01, 230 (2019) 0

9. Panda, M.: Major role of process conditions in tuning the percolation behavior of polyvinylidene fluoride based polymer/metal composites. Appl. Phys. Lett. 111, 082901 (2017) 0

10. Panda, M., Srinivas, V.and Thakur A. K. "Role of polymer matrix in large enhancement of dielectric constant in polymer-metal composites" Appl. Phys. Lett., 99, 042905 (2011)

11. Panda, M., Thakur, A.K.: and Srinivas V. "Thermal effects on the Percolation behavior of polyvinylidene fluoride/nickel composites". Journal of Appl. Polym. Sci. 117, 3023 ((2010), )

12. Panda, M., Srinivas, V.and A. K. Thakur "On the question of percolation threshold in polyvinylidene fluoride/nanocrystalline nickel composites" Appl. Phys. Lett. 92, 132905 (2008)

13. Panda, M., Srinivas, V., Thakur, A.K.: Surface and interfacial effect of filler particle on electrical properties of polyvinylidene fluoride/nickel composites. Appl. Phys. Lett. 93, 242908 ( (2008) "'”, ) 
14. Panda, M., and Trivedi, A.: Ferroelectric and Piezoelectric Properties of Cold Pressed Polyvinyledene fluoride/Barium Titanate Nano-composites. Ferroelectrics 572, 236 (2020) 0

15. Panda, M., Srinivas, V., and Thakur, A.K.: Multiferroic properties of PVDF/Ni nano composites. Ind. J. of Pure and Appl. Phys. 54, 144 (2016) 0

16. Méndez, S.L., Martins, P.: (2017). Magnetoelectric Polymer-Based Composites, Fundamentals and Applications. Wiley-VCH Verlag GmbH \& Co.KGaA, Boschstr. 12, 69469

17. Martins, P., Méndezc, S.L.: Polymer-based magnetoelectric materials: To be or not to be. Appl. Mat. Today 15, 558-561 (2019)

18. Chandrasekhar, K.D., Venimadhav, A., Das, A.K.: High dielectric permittivity in semiconducting Pr0.6Ca0.4MnO3Pr0.6Ca0.4MnO3 filled polyvinylidene fluoride nanocomposites with low percolation threshold. Appl. Phys. Lett. 95, 062904 (2009)

19. Srivastava, S., Haridas, M., Basu, J.K.: Optical properties of polymer nanocomposites. Bull. Mater. Sci. 31, 213-217 (2008)

20. Roppolo, I., Sangermano, M., Chiolerio, A.: Chapter 7: Optical Properties of Polymer Nanocomposites, Functional and Physical Properties of Polymer Nanocomposites. John Wiley \& Sons, Ltd. (2016)

21. Donya, H., Tahac, T.A., Alruwailic, A., Tomsahd, I.B.I., Ibrahimc, M.: Micro-structure and optical spectroscopy of PVA/iron oxide polymer nanocomposites. J. materres technol. 9, 9189-9194 (2020)

22. Rahman, Md.A., Chung, G.S.: Synthesis of PVDF-graphene nanocomposites and their properties. J. Alloy. Compd. 581, 724-730 (2013)

23. Mutlay, I., Tudoran, L.B.,. . Fullerenes: Percolation Behavior of Electrically Conductive Graphene Nanoplatelets/Polymer Nanocomposites:Theory and Experiment. Nanotubes, and Carbon Nanostructures 22, 413-433 (2014)

24. Panda, M., Sultana, N., Singh, A.K.: Structural and Optical properties of PVDF/GO nanocomposites, Fullerines, Nano tube and carbon nano structures, (2021) https://doi.org/10.1080/1536383X.2021.1966767

25. Hassan, D., Hashim, A.: Structural and Optical Properties of (Polystyrene-Copper Oxide) Nanocomposites for Biological Applications. J. Bionanosci. 12, 341-345 (2018)

26. Islam, A., Khan, A.N., Shakir, M.F., Islam, K.: Strengthening of $\beta$ polymorph in PVDF/FLG and PVDF/GO nanocomposites. Mater. Res. Exp. 7, 015017 (2020)

27. Patel, G., Sureshkumar, M.B., Patel, P.: Spectroscopic Investigation and Characterizations of PAM/PEO Blends Films. Soft 04, 02, 9-24 (2015)

28. Takahashi S., Okada H., Nobukawa S., Yamaguchi, M. Optical properties of polymer blends composed of poly(methyl methacrylate) and ethylene-vinyl acetate copolymer, European Polymer Journal Volume 48, Issue 5, May 2012, Pages 974-980.

29. Ahmed, H.T., Mohamad, J.V.J.TahirD.A., Abdullah, A.H.: O. G., 2019, Effect of PEG as a plasticizer on

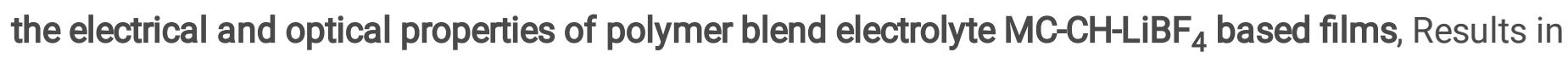
Physics, 15, 102735 
30. David M., Engel L., Diamand, Y. S. Spectroscopic ellipsometry study of spin coated P(VDF-TrFECTFE) thin films and P(VDF-TrFE-CTFE)/PMMA blends, Microelectronic Eng., 171, 2017, pp 37-43.

31. Schmiedova, V.; Heinrichova, P.; Zmeskal, O.; Weiter, M.; Characterization of polymeric thin films for photovoltaic applications by spectroscopic ellispometry Applied Surface Science, 349, 2015, pp 582588.

32. Lingjie, L., Jinglei, L., Liangliu, W., Fusheng, P.: 2021, Chapter 2 - Spectroscopic ellipsometry, in the book, Handbook of Modern Coating Technologies: Advanced Characterization Methods, pp 45-83, Edited by M. A. Khazraei, N. Ali, M. Chipara, N. Bensaada, L., Jeff; T.M. D., Hosson

33. Hummers, W.S., Offeman, R.E.: Preparation of Graphitic Oxide. J. Am. Chem. Soc. 80, 1339-1339 (1958)

\section{Tables}

Table 1. The details of the samples used in the preparation of PB with varying wt $\%$ of PEG.

\begin{tabular}{|c|c|c|c|c|c|}
\hline $\begin{array}{c}\text { Sample } \\
\text { Number }\end{array}$ & $\begin{array}{c}\text { Amount of } \\
\text { PVDF(g) }\end{array}$ & $\begin{array}{c}\text { Amount of } \\
\text { PEG(g) }\end{array}$ & $\begin{array}{c}\text { Amount of } \\
\text { Acetone(ml) }\end{array}$ & $\begin{array}{c}\text { Weight } \\
\text { Fraction of } \\
\text { PEG }\end{array}$ & $\begin{array}{c}\text { Percentage by Weight } \\
\text { of PEG (wt \%) }\end{array}$ \\
\hline 1 & 1.00 & 0.00 & 40 & 0.0 & 00 \\
\hline 2 & 0.90 & 0.10 & 40 & 0.1 & 10 \\
\hline 3 & 0.80 & 0.20 & 40 & 0.2 & 20 \\
\hline 4 & 0.70 & 0.30 & 40 & 0.3 & 30 \\
\hline 5 & 0.60 & 0.40 & 40 & 0.4 & 40 \\
\hline
\end{tabular}

Table 2. The details of the samples used in the preparation of PNC with varying wt\% of GO. 


\begin{tabular}{|c|c|c|c|c|c|}
\hline $\begin{array}{l}\text { Sample } \\
\text { Number }\end{array}$ & $\begin{array}{l}\text { Amount of } \\
\text { PVDF(g) }\end{array}$ & $\begin{array}{l}\text { Amount of } \\
\text { GO(g) }\end{array}$ & $\begin{array}{l}\text { Amount of } \\
\text { Acetone(ml) }\end{array}$ & $\begin{array}{l}\text { Weight } \\
\text { Fraction of GO }\end{array}$ & $\begin{array}{l}\text { Percentage beight of GO(wt\%) } \\
\text { Wrot }\end{array}$ \\
\hline 1 & 0.5 & 0.00 & 25 & 0.0 & 0 \\
\hline 2 & 0.5 & 0.005 & 25 & 0.01 & 2 \\
\hline 3 & 0.5 & 0.01 & 25 & 0.02 & 3 \\
\hline 4 & 0.5 & 0.015 & 25 & 0.03 & 5 \\
\hline 5 & 0.5 & 0.025 & 25 & 0.05 & 10 \\
\hline 6 & 0.5 & 0.05 & 25 & 0.10 & 20 \\
\hline 7 & 0.5 & 0.1 & 25 & 0.20 & 2 \\
\hline
\end{tabular}

Figures 


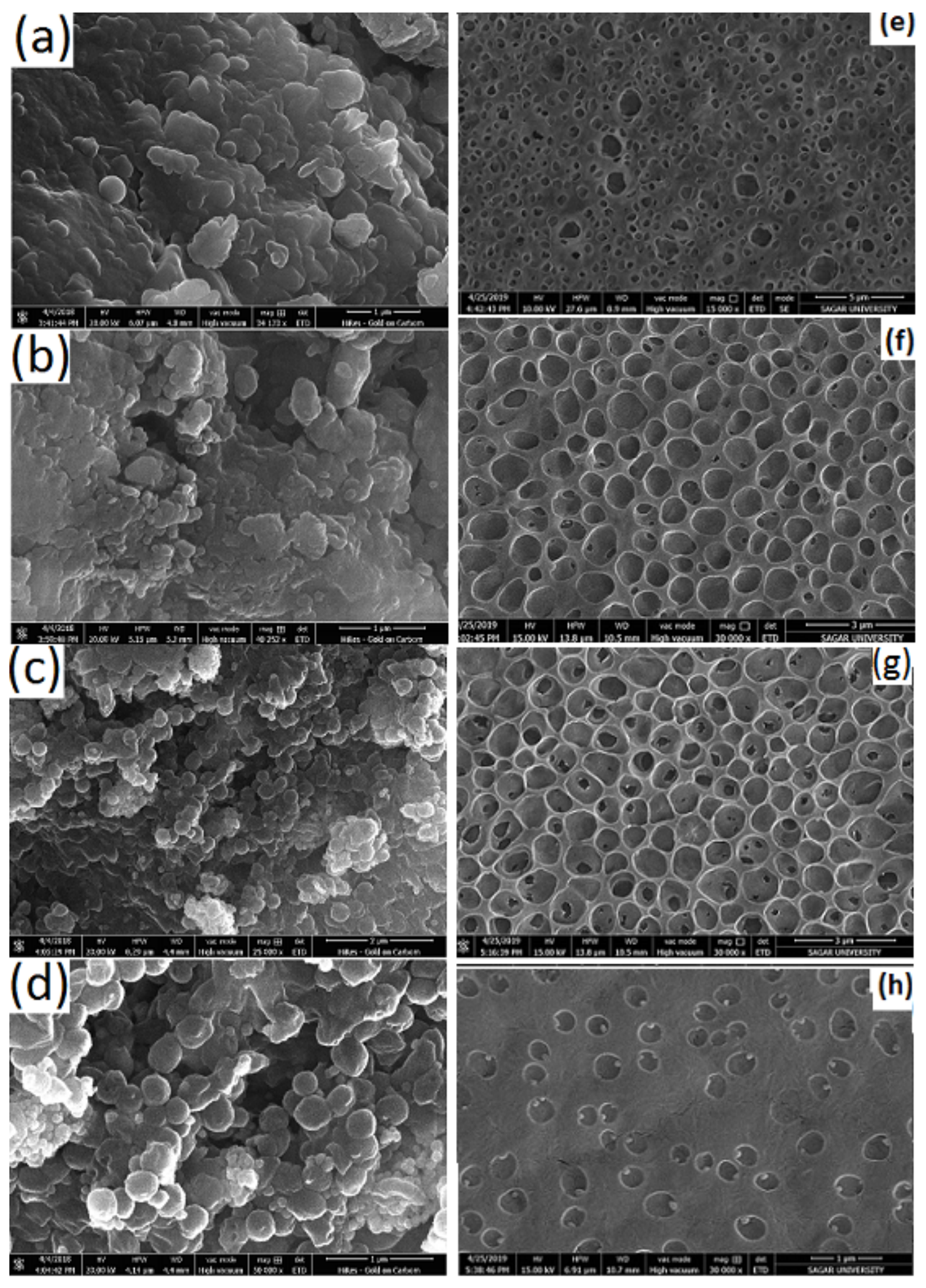

\section{Figure 1}

Scanning Electron Micrographs of PVDF/PEG PB with varying weight\% of PEG (a) $0 \%$ (b) $20 \%$ (c) $40 \%$ [lower resolution] \& (d) $40 \mathrm{wt} \%$ [higher resolution] and for PVDF/GO PNC with varying weight\% of GO (e) $2 \%$ (f) $5 \%$ (g) $10 \%$ \& (h) $20 \mathrm{wt} \%$, respectively. 


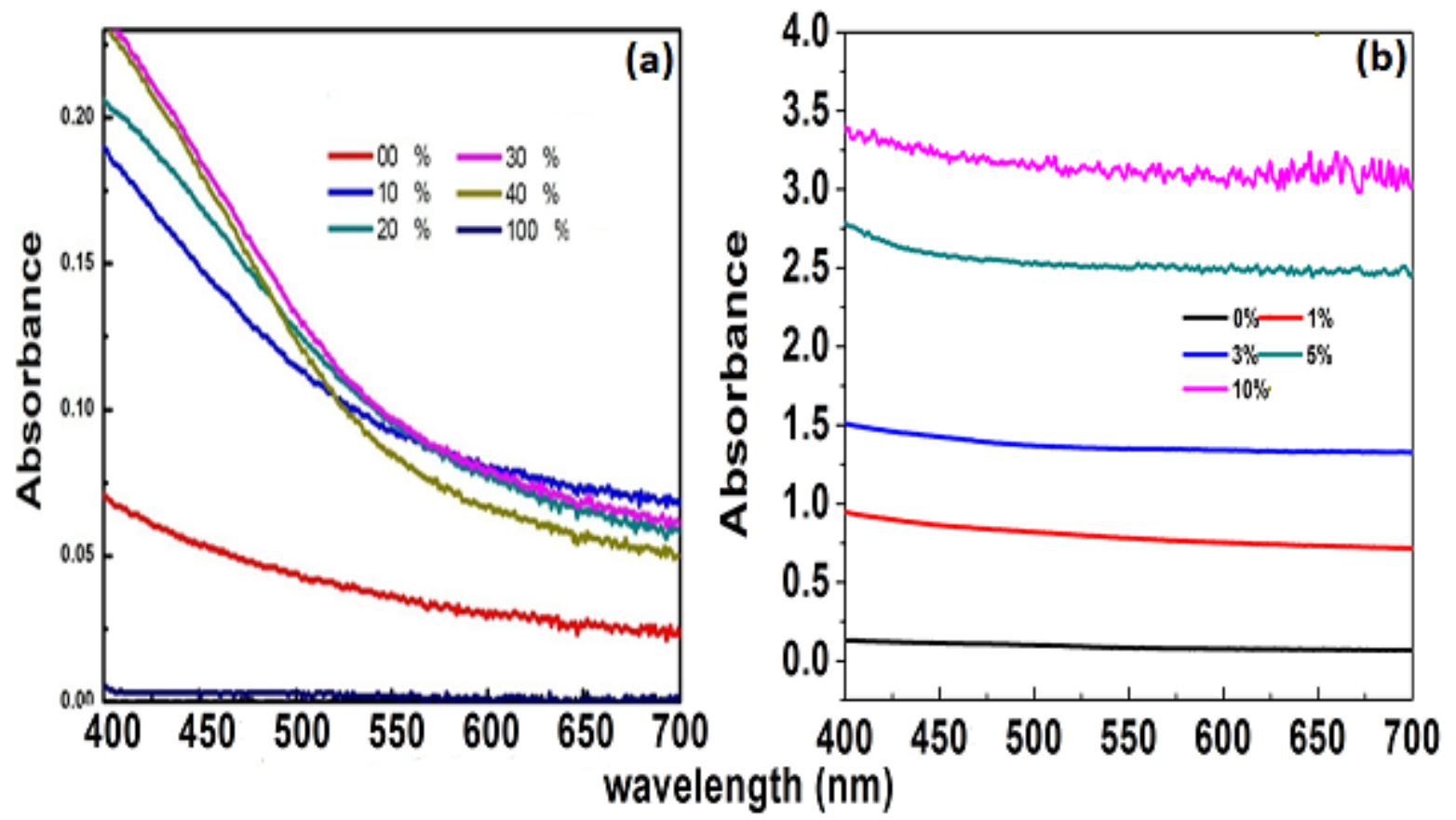

Figure 2

Variation of absorbance with wavelength with varying weight \% of their respective fillers (a) PVDF/PEG PB and (b) PVDF/GO PNC, respectively.
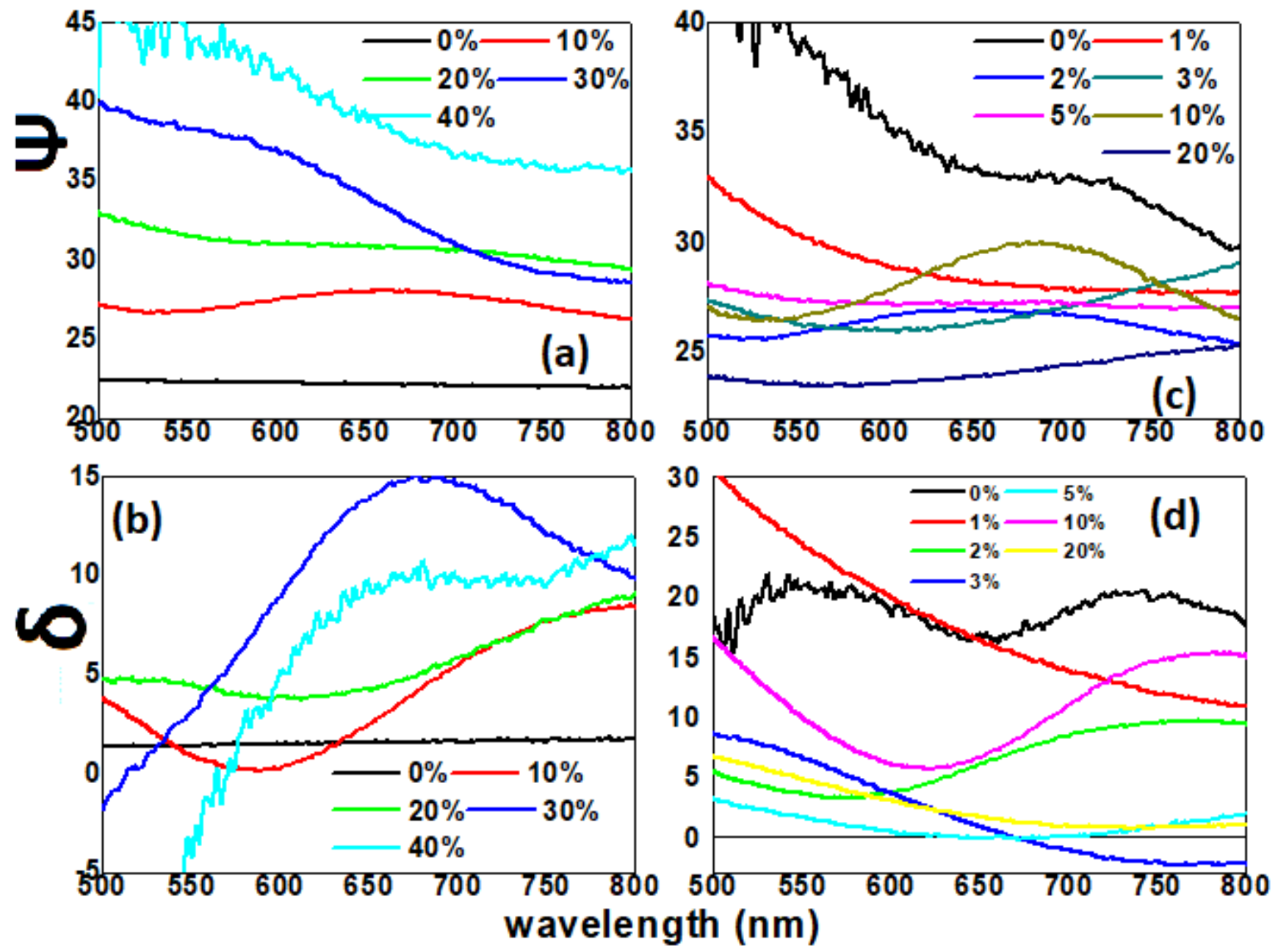
Figure 3

Variation of real and imaginary parts of polarization parameter with wavelength for PVDF/PEG PB (a) Psi $(\psi)(b)$ Delta ( $)$ and for PVDF/GO PNC (c) Psi ( $\psi)(d)$ Delta, respectively.
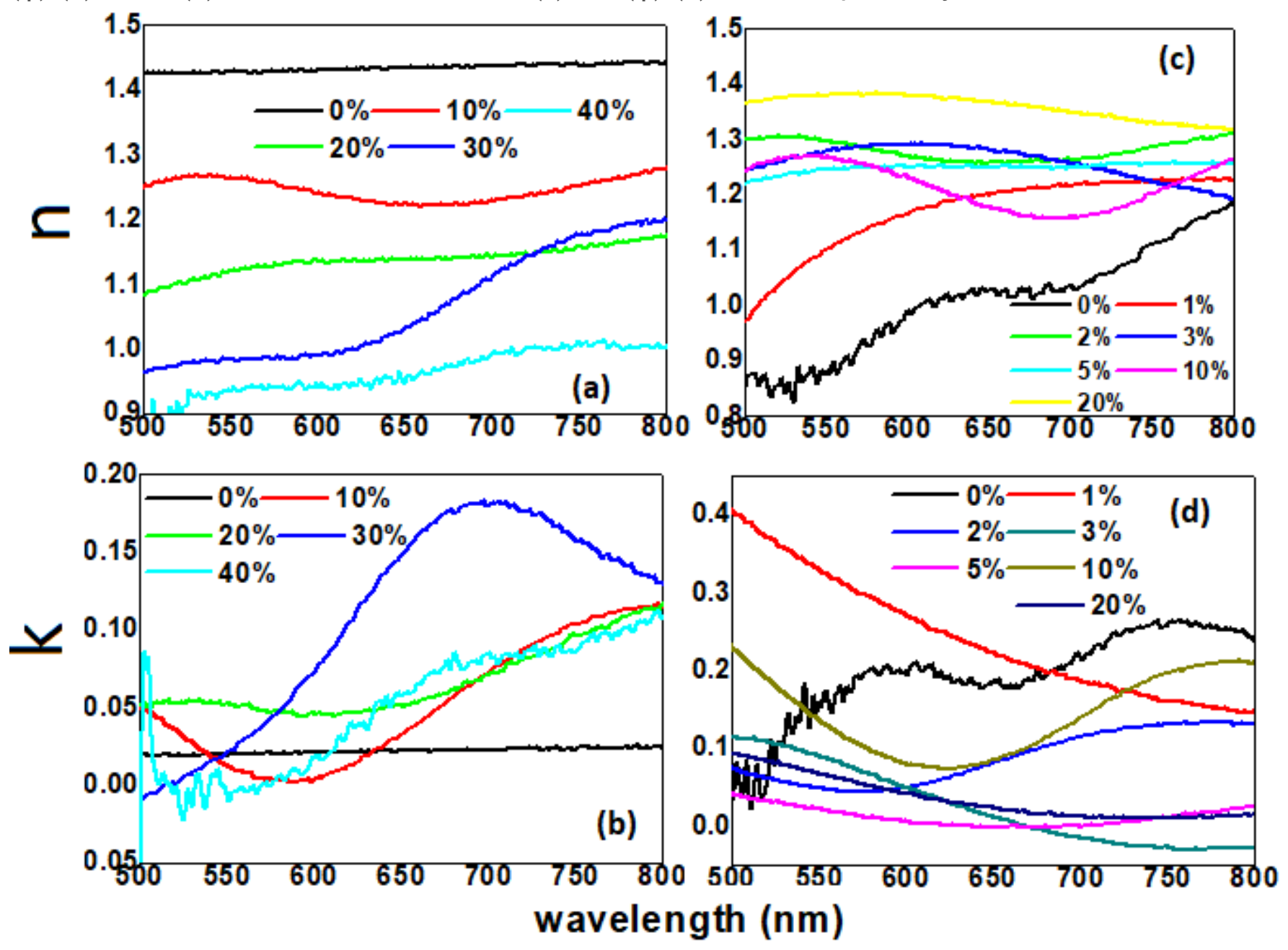

Figure 4

Variation of real and imaginary parts of refractive index with wavelength for PVDF/PEG PB (a)real part of refractive index (n) (b) imaginary part of refractive index (k) and for PVDF/GO PNC(c) real part of refractive index $(n)(d)$ imaginary part of refractive index $(k)$, respectively. 

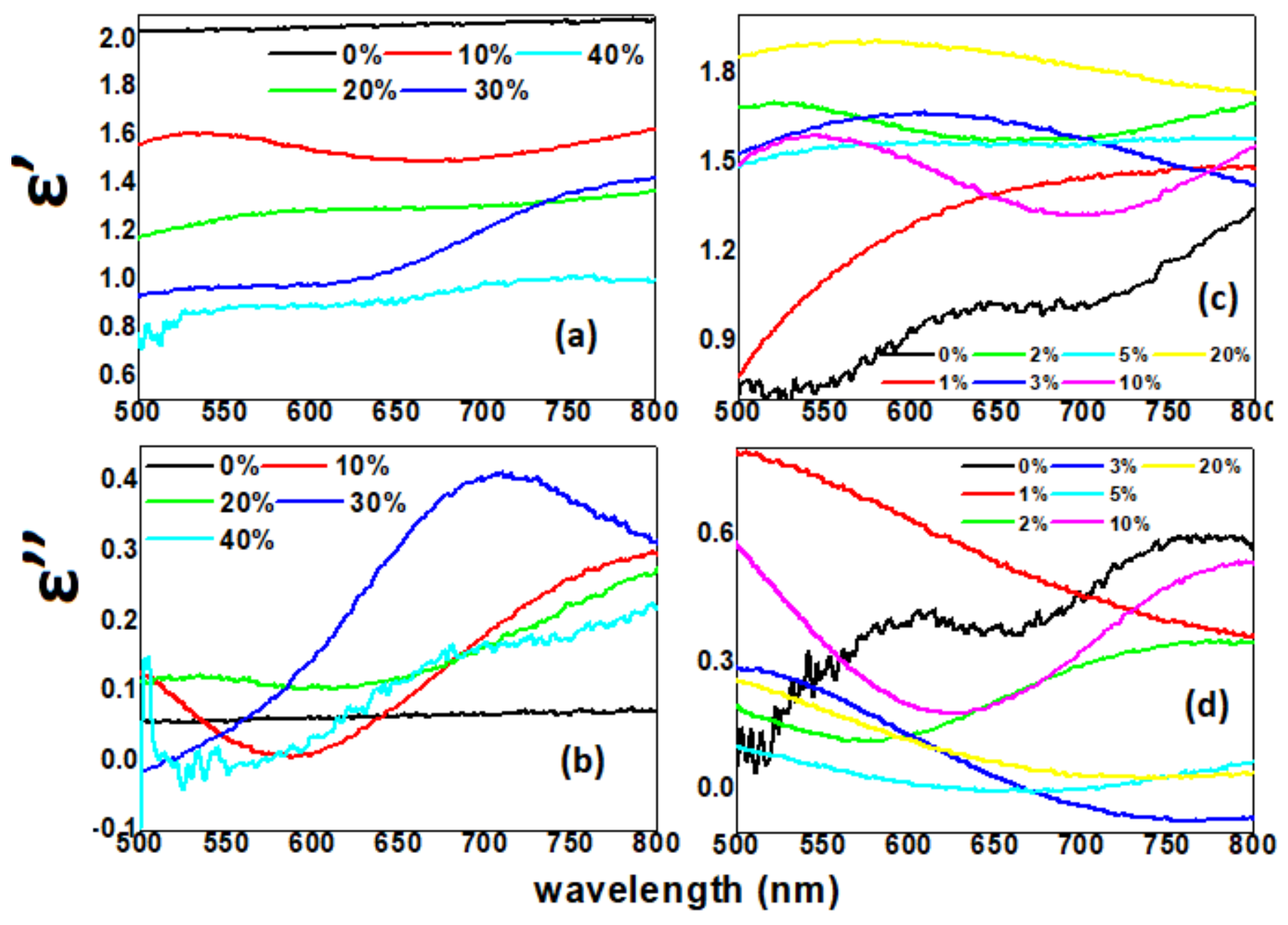

Figure 5

Variation of real and imaginary parts of complex dielectric function with wavelength for PVDF/PEG PB (a)real part of complex dielectric function $\left(\varepsilon^{\prime}\right)(b)$ imaginary part of complex dielectric function $\left(\varepsilon^{\prime \prime}\right)$ and for PVDF/GO PNC (c)real part of complex dielectric function $\left(\varepsilon^{\prime}\right)(d)$ imaginary part of complex dielectric function $(\varepsilon ")$, respectively. 